\title{
microRNA-216b enhances cisplatin-induced apoptosis in osteosarcoma MG63 and SaOS-2 cells by binding to JMJD2C and regulating the HIF1a/HES1 signaling axis
}

Dong Yang, Tianyang Xu, Lin Fan, Kaiyuan Liu and Guodong Li

\begin{abstract}
Background: Although cisplatin-based chemotherapy represents the standard regimen for osteosarcoma (OS), OS patients often exhibit treatment failure and poor prognosis due to chemoresistance to cisplatin. Emerging research has highlighted the tumor suppressive properties of microRNAs (miRNAs or miRs) in various human cancers via the inhibition of the histone demethylase jumonji domain containing protein 2C (JMJD2C). As a coactivator for hypoxiainducible factor 1a (HIF1a), JMJD2C targets hairy and enhancer of split-1 (HES1) gene. Hence, the current study aimed to elucidate the role of miR-216b in OS cell cisplatin resistance to identify the underlying mechanism of miR216b regulating the JMJD2C//HIF1a/HES1 signaling.
\end{abstract}

Methods: Tumor and paracancerous tissues were collected from OS patients to determine the expression patterns of miR-216b and JMJD2C. After ectopic expression and knockdown experiments in the OS cells, CCK-8 assay and flow cytometry were employed to determine cell viability and apoptosis. The interaction of miR-216b, JMJD2C, HIF1a and HES1 was subsequently determined by dual luciferase reporter, co-immunoprecipitation (IP) and ChIPqPCR assays. In vivo experiments were conducted to further verify the role of the miR-216b in the resistance of OS cells to cisplatin.

Results: miR-216b expression was reduced in the OS tissues, as well as the MG63 and SaOS-2 cells. Heightened miR-216b expression was found to be positively correlated with patient survival, and miR-216b further enhanced cisplatin-induced apoptosis of MG63 and SaOS-2 cells. Mechanistically, miR-216b inhibited JMJD2C expression by binding to its $3^{\prime} \mathrm{UTR}$. Through interaction with HIF1a, JMJD2C removed the H3K9 methylation modification at the HES1 promoter region, leading to upregulation of HES1 in vitro. Furthermore, miR-216b was observed to increase the tumor growth in nude mice in the presence of cisplatin treatment. HES1 overexpression weakened the effects of miR-216b in MG63 and SaOS-2 cells and in nude mouse xenografts.

Conclusion: Overall, miR-216b enhanced the sensitivity of OS cells to cisplatin via downregulation of the JMJD2C/ HIF1a/HES1 signaling axis, highlighting the capacity of miR-216b as an adjunct to cisplatin chemotherapy in the treatment of OS.

Keywords: microRNA-216b, JMJD2C, HIF1a, HES1, Osteosarcoma, Cisplatin

\footnotetext{
* Correspondence: zwcqdsl@163.com

Department of Orthopedics, Shanghai Tenth People's Hospital, Tongji

University School of Medicine, Shanghai 200433, P.R. China
}

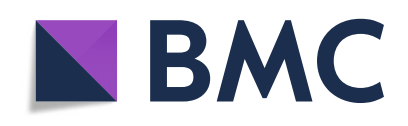

(- The Author(s). 2020, corrected publication [2020]. Open Access This article is licensed under a Creative Commons Attribution 4.0 International License, which permits use, sharing, adaptation, distribution and reproduction in any medium or format, as long as you give appropriate credit to the original author(s) and the source, provide a link to the Creative Commons licence, and indicate if changes were made. The images or other third party material in this article are included in the article's Creative Commons licence, unless indicated otherwise in a credit line to the material. If material is not included in the article's Creative Commons licence and your intended use is not permitted by statutory regulation or exceeds the permitted use, you will need to obtain permission directly from the copyright holder. To view a copy of this licence, visit http://creativecommons.org/licenses/by/4.0/. The Creative Commons Public Domain Dedication waiver (http:// creativecommons.org/publicdomain/zero/1.0/) applies to the data made available in this article, unless otherwise stated in a credit line to the data. 


\section{Background}

Osteosarcoma (OS) represents one of the most common primary bone malignancies, predominantly affecting children and adolescents [1]. In addition to the highest incidence of OS in adolescence, a second incidence peak has been reported in the elderly, a high-risk population group [2]. The incidence of OS is estimated as ' 4 ' for the $0-14$ years age range and ' 5 ' for 15-19 years age range per million people worldwide [3]. Moreover, the 5-year survival rate worldwide for OS remains to be largely appalling, with studies estimating a survival rate between 40 and $70 \%[3,4]$, a statistic which has failed to improve for decades [5]. Young age, first primary tumor, localized stage, low grade, and surgical treatment are factors that have all been positively linked with overall survival in OS [6]. Currently, a combination of surgical intervention as well as chemotherapy is regarded as the first line treatment for OS. However, it is well-documented that the advent of chemotherapy resistance is a big obstacle in the treatment of OS [7-10], which may, at least partly, contribute to the unfavorable outcomes seen in OS patients. Hence, the current study set out to identify the potential mechanism by which chemotherapy drug resistance occurs during the course of OS treatment.

microRNAs (miRNAs or miRs) are known to possess the ability to regulate cell proliferation and apoptosis, and as a result, are implicated in a wide variety of human cancers as oncogenes or tumor suppressors [11]. MiRNAs refer to small non-coding RNA molecules, which serve as regulators of protein expression via the degradation of targeted mRNA or blockade of protein translation [12]. More notably, one such miRNA, miR$216 \mathrm{~b}$ has been recently shown to potentially exert a tumor inhibitor function in OS by targeting FoxM1 as it inhibits OS cell proliferation, migration, and invasion [13]. Moreover, the over-expression of miR-216b has also been previously demonstrated to significantly enhance the sensitivity of non-small cell lung cancer cells to cisplatin-induced apoptosis by targeting c-Jun [14]. In addition, miR-216b can diminish cell proliferation, while promoting the sensitivity of colorectal cancer cells to oxaliplation by suppressing PDZ-binding kinase [15]. These findings lead the authors to hypothesize that miR216b may confer a similar role in OS to affect the chemosensitivity of OS cells.

Histone demethylase jumonji $\mathrm{C}$ domain-containing $2 \mathrm{C}$ (JMJD2C) is known to demonstrate regulatory potentials in the epigenetic mechanism in malignant diseases, particularly in regard to moderating the influence on the promoter activity of target genes which are strongly associated with tumor development [16]. Prior evidence has proposed that JMJD2C is highly-expressed in OS, and even confers a regulatory role in the context of OS [17]. Moreover, JMJD2C has also been shown to serve as a co-activator for hypoxia-inducible factor 1 (HIF1 $\alpha$ ) for cancer progression [18]. Meanwhile, as a widelydocumented regulator of cellular metabolism, HIF1 $\alpha$ performs essential functions in the survival and differentiation of mesenchymal stem cells [19]. HIF1 $\alpha$ has been reported to be highly-expressed in OS [20], and to further function to activate downstream genes through its transcriptional activation to promote OS [21]. More importantly, HIF1 $\alpha$ has been reported to induce drug resistance through the hairy and enhancer of split-1 gene (HES1) in breast cancer [22]. HES1 represents a critical factor for the maintenance of stem cells, quiescent cells or cancer cells, and has also been demonstrated to elicit drug resistance and metastasis of tumor cells [23]. Furthermore, HES1 has been reported to be highly-active in OS [24], with studies suggesting that HES1 promotes the critical phenomenon of chemotherapy resistance in cancer $[25,26]$. In order to effectively inhibit HES1 to reduce chemotherapy resistance, one method is to modify H3K9 methylation at its gene promoter [27]. Hence, the current study set out to examine whether miR-216b inhibits cancer growth in OS by reducing the levels of JMJD2C and HES1. We further aimed to determine the involvement of HIF1 $\alpha$ and H3K9 methylation modification in the underlying mechanism associated with the effects of miR-216b.

\section{Materials and methods}

\section{Ethics statement}

The current study was performed with the approval of the Ethics committee of Shanghai Tenth People's Hospital (approval number: 2010-0017) and in strict accordance with the Declaration of Helsinki. Signed informed consents were obtained from all participants or their guardians prior to the study. Animal experiments were performed according to a strictly designed protocol, in accordance with the Guide for the Care and Use of Laboratory Animals published by the US National Institutes of Health. All efforts were made to ensure minimal suffering of the animal included in the study.

\section{Sample collection from OS patients}

Cancer tissues and paracancerous tissues were collected from a total of 60 OS patients (aged 10-58 years, with an average age of $21.63 \pm 9.70$ years old) at the Shanghai Tenth People's Hospital from April 2010 to April 2013. All included patients had not undergone chemotherapy or radiotherapy prior to specimen collection. Patient follow-up was conducted over a period of 60 months. The time from specimen collection to tumor recurrence or death (overall survival) was recorded. The last followup date was recorded if no tumor recurrence or death occurred. All included patients received the same treatment regimen at the Shanghai Tenth People's Hospital, 
with no metastasis occurring during the follow-up period.

\section{Cell transfection}

Human osteosarcoma cell lines U2OS, HOS, SaOS-2, MG63 and human embryonic immortalized osteoblasts hFOB1.19 cells (American Type Culture Collection, Manassas, VA, USA) were cultured in Dulbecco's modified Eagle's medium (DMEM; HyClone, Logan, UT USA) supplemented with $10 \%$ fetal bovine serum, $100 \mu \mathrm{g} / \mathrm{mL}$ streptomycin, and $100 \mathrm{U} / \mathrm{mL}$ penicillin at $37^{\circ} \mathrm{C}$ with $5 \% \mathrm{CO}_{2}$ [28].

The MG63 and SaOS-2 cells were subsequently transfected with agomir negative control (NC), miR-216b agomir, agomir NC + over-expression (oe)-NC, miR-216b agomir + oe-NC, miR-216b agomir + oe-JMJD2C, miR-216b agomir + oe-HES1, oe-NC + lentivirus (LV)-short hairpin RNA (sh)NC, oe-JMJD2C + LV-shNC, oe-JMJD2C + LV-shHIF1 $\alpha$, LV-shJMJD2C + oe-NC, and LV-shJMJD2C + oe-HIF1 $\alpha$. The cDNA constructs (pcDNA3.1-NC, pcDNA3.1-JMJD2C, pcDNA3.1-HIF1 $\alpha$ and pcDNA3.1-HES1) were all constructed by HanBio Biotechnology Co., Ltd. (Shanghai, China). Meanwhile, agomir NC (5'-UUCUCCGAACGUGU CACGUTT-3'), miR-216b agomir (5'-AAAUCUCUGCAG GCAAAUGUGA-3'), LV-shNC, LV-shHIF1 $\alpha, \quad$ LVshJMJD2C were all designed and constructed by GenePharma (Shanghai, China). Transfection was performed using Lipofectamine 2000 reagents (Invitrogen, Carlsbad, CA, USA) in accordance with the manufactures' instructions. JMJD2C (gene ID: 23081), HIF1 (gene ID: 3091) and HES1 (gene ID: 3280) were identified by the National Center for Biotechnology Information (NCBI) database.

\section{RNA quantitation by reverse transcription quantitative polymerase chain reaction (RT-qPCR)}

Total RNA content was extracted from the fresh tissues using RNeasy Mini kits (Qiagen, Valencia, CA, USA). For mRNA detection, the extracted total RNA was reverse transcribed into complementary DNA (cDNA) with the help of reverse transcription kits (RR047A, Takara, Japan). For miRNA detection, cDNA was obtained using miRNA First Strand cDNA Synthesis (Tailing Reaction) kits (B532451-0020, Sangon Biotech Co., Ltd., Shanghai, China). The samples were then loaded using a SYBR ${ }^{\bullet}$ Premix Ex TaqTM II (Perfect Real Time) kit (DRR081, Takara, Japan). RT-qPCR was performed on an ABI 7500 instrument (Applied Biosystems, Foster City, CA, USA), and each sample was evaluated in triplicate. The universal negative primers for miRNA, while the internal reference U6 were provided by miRNA First Strand cDNA Synthesis (Tailing Reaction) kit. The remaining primers were provided by Sangon Biotech Co., Ltd. (Shanghai, China). The primer sequences are depicted in Table 1 . The Ct value of each target gene was recorded and normalized to an internal reference, namely, $\beta$-actin or U6. Relative expression of all target genes was calculated by means of relative quantification $\left(2^{-\Delta \Delta \mathrm{Ct}}\right.$ method) [29].

\section{Protein expression determined by western blot analysis}

Total protein content was extracted from the tissues or cells using radioimmunoprecipitation assay (RIPA) lysis buffer containing phenylmethylsulphonyl fluoride (PMSF) on ice for $30 \mathrm{~min}$, followed by centrifugation at $8000 \mathrm{~g}$ and $4{ }^{\circ} \mathrm{C}$ for $10 \mathrm{~min}$. The supernatant was then collected for protein concentration determination using a bicinchoninic acid (BCA) protein assay kit (Thermo Fisher Scientific, Rockford, IL, USA, \#23250). Protein $(50 \mu \mathrm{g})$ was subsequently dissolved in $2 \times$ sodium dodecyl sulfate (SDS) loading buffer, boiled at $100^{\circ} \mathrm{C}$ for 5 min, and then separated using SDS-polyacrylamide gel electrophoresis (PAGE). The protein was subsequently transferred onto polyvinylidene fluoride (PVDF) membranes, which were blocked by $5 \%$ skim milk at room temperature for $1 \mathrm{~h}$ and incubated with primary rabbit antibodies against JMJD2C (ab85454, dilution ratio of 1: 1000), HIF1 $\alpha$ (ab51608, dilution ratio of 1:500), HES1 (ab71559, dilution ratio of 1:500), Ki67 (ab92742, dilution ratio of 1:5000), B-cell lymphoma 2 (Bcl-2) (ab32124, dilution ratio of 1:1000), and $\beta$-actin (ab227387, dilution ratio of 1:5000) overnight at $4{ }^{\circ} \mathrm{C}$. After three Tris-buffered saline Tween-20 (TBST) washes (10 min per wash), the membranes were then incubated with horseradish peroxidase (HRP)-labeled secondary goat anti-rabbit immunoglobulin G (IgG) H\&L (ab97051, dilution ratio of 1:2000) for $1 \mathrm{~h}$. All the aforementioned antibodies were purchased from Abcam Inc. (Cambridge, UK). The immunocomplexes on the membrane were visualized using enhanced chemiluminescence (ECL) reagents (BB-3501, Amersham, UK), with the band intensities quantified with the Bio-Rad Image Analysis System (Bio-Rad, Inc., Hercules, CA, USA), and Quantity One v4.6.2 software. The ratio of the gray value of the target band to $\beta$-actin was regarded as a reflection of the relative protein expression.

Table 1 Primer sequences for RT-qPCR

\begin{tabular}{ll}
\hline Target & Primer sequence (5'-3') \\
\hline miR-216b & Forward: 5'-GCCGCGCTAAAGTGCTTATAGTG-3' \\
HIF-1a & Reverse: 5'-CACCAGGGTCCGAGGT-3' \\
B-actin & Forward: 5'-CAGAAGATACAAGTAGCCTC-3' \\
& Reverse: 5'-CTGCTGGAATACTGTAACTG-3' \\
& Forward: 5'-GCGAGAAGATGACCCAGGATC-3' \\
& Reverse: 5'-CCAGTGGTACGGCCAGAGG-3' \\
U6 & Forward: 5'-ACGCTTCACGAATTGCGTGTC-3' \\
& Reverse: 5'-GCTTCGGCAGCACATATACTAAAAT-3'
\end{tabular}




\section{Binding relationship determined by dual luciferase reporter gene assay}

Wild type (wt) (pGL3-wt-JMJD2C-3'untranslated region [UTR]) or mutant (mut) (pGL3-mut-JMJD2C-3'UTR) reporter plasmids (GenePharma, Shanghai, China) were co-transfected with agomir NC or miR-216b agomir into MG63 and SaOS-2 cells. Following a $48 \mathrm{~h}$ period of transfection, the cells were collected and lysed. The subsequent procedures were performed with using a luciferase assay kit (K801-200, Biovision, Milpitas, CA, USA) following the manufacturer's instructions on the luciferase reporter assay system (Promega, Madison, WI, USA). The relative luciferase activity was calculated based on the ratio of the luciferase activity of firefly luciferase to that of renilla luciferase. The sequences of wt-JMJD2C3'UTR and mut-JMJD2C-3'UTR were 5'-GCAUGU AUGCUAAUGAGAUUU-3' and 5'-GCUGUAAACG ACGUCUCUAAA-3', respectively [30].

\section{Binding of JMJD2C to HIF1a determined by co- immunoprecipitation (co-IP) assay}

The cells were lysed for $30 \mathrm{~min}$ at $4{ }^{\circ} \mathrm{C}$ in RIPA buffer (Thermo Scientific, Waltham, MA, USA), and then centrifuged at $13,000 \mathrm{~g}$ for $30 \mathrm{~min}$ at $4{ }^{\circ} \mathrm{C}$. The supernatant was subsequently collected and incubated with specific antibody at $4{ }^{\circ} \mathrm{C}$ overnight, followed by the addition of pierce protein A/G Magnetic Beads (\#88803, Thermo Scientific) and $4 \mathrm{~h}$ of incubation by shaking at $4{ }^{\circ} \mathrm{C}$. The beads were then centrifuged, washed three times, and mixed with the loading buffer, followed by protein evaluation using SDS-PAGE and immunoblotting [31].

\section{Quantity of the HES1 promoter determined by chromatin immunoprecipitation (ChIP)-qPCR}

ChIP was performed using EZ-Magna ChIP A/G Chromatin Immunoprecipitation kits (17-371, Millipore, Billerica, MA, USA). Briefly, the cells were sonicated and centrifuged at $12,000 \mathrm{~g}$ for $10 \mathrm{~min}$ at $4{ }^{\circ} \mathrm{C}$ to remove insoluble precipitates. The cells were subsequently incubated with protein $\mathrm{G}$ agarose beads at $4{ }^{\circ} \mathrm{C}$ for $1 \mathrm{~h}$, and centrifuged at $5000 \mathrm{~g}$ for a total of $1 \mathrm{~min}$. Next, $10 \mu \mathrm{L}$ (1\%) of the supernatant was collected as the 'Input', while the remaining supernatant was divided into 2 portions and incubated with antibodies against H3K9me3 (ab8898, dilution ratio of 1:20, Abcam Inc., Cambridge, UK), H3K9me2 (ab1220, dilution ratio of 1:10, Abcam Inc., Cambridge, UK), H3 (ab213257, dilution ratio of 1: 5, Abcam Inc., Cambridge, UK), and NC rabbit antihuman IgG (ab2410, dilution ratio of 1:25, Abcam Inc., Cambridge, UK) at $4{ }^{\circ} \mathrm{C}$ overnight. The precipitated protein-DNA complex was then incubated with protein $\mathrm{G}$ agarose beads for $1 \mathrm{~h}$ at $4{ }^{\circ} \mathrm{C}$. After centrifugation at $5000 \mathrm{~g}$ for $1 \mathrm{~min}$, the supernatant was discarded. The protein-DNA complex was fragmented overnight at $65^{\circ} \mathrm{C}$. The DNA fragment was recovered and used as an amplification template for RT-qPCR. The primers employed to detect the quantity of HES1 promoter were agomir NC (5'-UUCUCCGAACGUGUCACGUTT-3') and miR-216b agomir ( $5^{\prime}$-AAAUCUCUGCAGGCAAAU GUGA-3').

\section{Cell viability assays}

Cell proliferation was measured using a cell counting kit-8 (CCK-8) assay (Kumamoto, Japan). MG63 and SaOS-2 cells were firstly seeded in 96-well plates. After $24 \mathrm{~h}$ of transfection, various concentrations of cisplatin $(0,0.2,0.4,0.8,1.6 \mu \mathrm{M})$ were added to the cells and incubated for $72 \mathrm{~h}$. The CCK- 8 reagent $(10 \mu \mathrm{L})$ was then added to each well and incubated for $1 \mathrm{~h}$. Absorbance was finally measured at a wavelength of $450 \mathrm{~nm}$ using a microplate reader.

\section{Apoptosis assays}

After $24 \mathrm{~h}$ of transfection, the cells were treated with $1 \mu \mathrm{M}$ cisplatin [32] for $72 \mathrm{~h}$. An apoptosis assay was subsequently performed using Annexin V-fluorescein isothiocyanate/propidium iodide (FITC/PI) kits (KeyGEN Biotech. Co., Ltd., Nanjing, China). The cells were subsequently observed and analyzed using a flow cytometer (FACSCalibur, BD Biosciences, Franklin Lakes, NY, USA). The experiment was performed in triplicate, with the respective average values obtained and recorded.

\section{Tumor xenograft in nude mice}

A total of $30 \mathrm{BALB} / \mathrm{c}$ nude mice (aged 5-6 weeks old, weighing 15-18 g, acquired from the Shanghai Experimental Animal Center of Chinese Academy of Sciences) were randomly divided into the 5 following groups (6 mice per group): control group, Lv-oe-NC group, Lv-oe- $\mathrm{NC}+$ Cis group, Lv-oe-miR-216b $+\mathrm{Lv}-\mathrm{oe}-\mathrm{NC}+\mathrm{Cis}$ group, and Lv-oe-miR-216b + Lv-oe-HES1 + Cis group. The lentiviruses carrying oe-NC, oe-miR-216b and oe-HES1 were purchased from Shanghai Gene Pharma Co., Ltd. (Shanghai, China). Following lentivirus infection, the stablytransfected MG63 cells were subcutaneously injected into the axillary region of nude mice at a density of $1 \times 10^{6}$ cells. From the 7 th day of inoculation, cisplatin $(8 \mathrm{mg} / \mathrm{kg}$, [33]) was intraperitoneally administered to the mice in the Lv-oe-NC + Cis, Lv-oe-miR-216b + Lv-oe-NC + Cis, and Lv-oe-miR-216b + Lv-oe-HES1 + Cis groups twice a week. On the 14th day, the mice were euthanized using carbon dioxide. The tumors were subsequently removed, after which the tumor volume was measured using the following formula: $\left(a \times b^{2}\right) / 2$ [34], where a represented the length of the tumor and $b$ was the width of the tumor. The volume of the tumor was calculated and a tumor growth curve was then plotted [35]. 


\section{Statistical analysis}

Data analyses were processed using the SPSS 21.0 statistical software (IBM Corp. Armonk, NY, USA). Measurement data were expressed as mean \pm standard deviation. Data with normal distribution and homogeneity of variance between two groups were compared using paired $t$ test (paired data) or unpaired $t$-test (unpaired data). Data comparisons between multiple groups were performed using one-way analysis of variance (ANOVA), followed by a Tukey's test. Data comparison between groups at different time points was performed by repeated measures ANOVA with Bonferroni post-hoc test. Correlation of two variants was analyzed by Pearson correlation coefficient. Patient survival rate was analyzed using the Kaplan-Meier method followed by log-rank test. A $p<0.05$ value was considered to be indicative of statistical significance.

\section{Results}

miR-216b is poorly-expressed in OS and its high expression is positively-correlated with patient survival Firstly, the expression of miR-216b was found to be markedly lower in OS tissues compared to that in paracancerous tissues (fold $=0.315$ ) (Fig. 1a). The median miR-216b relative expression pattern in cancer tissues from 60 patients was subsequently determined, after which the patients with values above the median were placed into the high miR-216b expression group, while those below the median were regarded as the low miR-216b expression group. In addition, Kaplan-Meier survival analysis revealed that elevated expression of miR-216b was correlated with higher overall survival in OS patients (Fig. 1b). However, no significant correlation was identified between the miR$216 \mathrm{~b}$ expression and factors such as age, sex, location of the tumor, and metastasis (Table 2). Additionally, results of multivariate analysis demonstrated that miR-216b served as an independent prognostic factor for OS (Table 3). Lastly, the expression of miR-216b was determined to be significantly lower in the MG63 and SaOS-2 cell lines relative to other OS cell lines and control (hFOB1.19 cell line) (U2OS: fold $=0.436$, HOS: fold $=$ 0.614, SaOS-2: fold =0.248, MG63: fold =0.161) (Fig. 1c). Hence, MG63 and SaOS-2 cells were selected for subsequent experimentation.

\section{miR-216b improves the effects of cisplatin on OS cells in vitro}

We subsequently set out to evaluate the sensitivity of MG63 and SaOS-2 cells to cisplatin as OS resistance against chemotherapy remains a significant obstacle during the treatment of OS. Transfection of miR-216b agomir, as expected, elevated the expression of miR-216b in MG63 and SaOS-2 cells, indicating successful transfection (MG63: fold = 2.218, SaOS-2: fold = 3.094) (Supplementary Fig. 1). Meanwhile, it was found that cisplatin decreased the cell viability in MG-63 and SaOS-2 cells but much more so in cells transfected with miR-216b agomir (MG63: 0.25 fold $=0.73,0.5$ fold $=0.64,1$ fold $=$ 0.39; SaOS-2: 0.25 fold $=0.77,0.5$ fold $=0.72,1$ fold $=$ 0.46) (Fig. 2a). Cisplatin was found to also augment cell apoptosis, which was further elevated following miR216b agomir treatment in cells (MG63: miR-216b agomir fold $=1.39$; SaOS-2: $\mathrm{miR}-216 \mathrm{~b}$ agomir fold $=1.26$ ) (Fig. 2b, c). These results suggested that miR-216b enhanced the promotive effects of cisplatin on OS cell apoptosis, while inhibiting cell viability in vitro.

\section{miR-216b targets and inhibits JMJD2C to enhance cisplatin-induced apoptosis in OS cells}

The online bioinformatic website Starbase [36] predicted the presence of binding sites of miR-216b to the 3'UTR of the histone demethylase JMJD2C mRNA (KDM4C). Subsequently, a dual luciferase reporter assay was performed, which revealed that co-transfection of miR-216b agomir with wt-JMJD2C-3'UTR triggered a reduction in the luminescence intensity (MG63 fold =0.407; SaOS-2 fold $=0.442$ ), whereas no alterations were detected upon co-transfection with mut-JMJD2C-3'UTR in cells (MG63
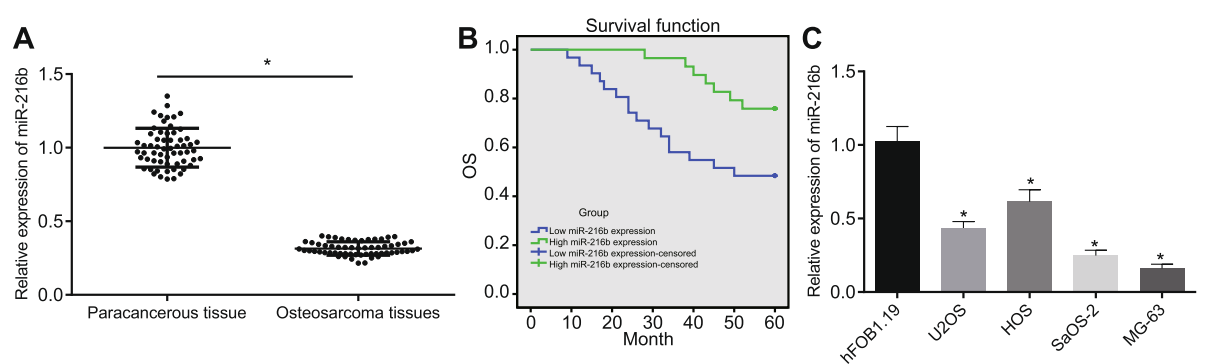

Fig. 1 miR-216b expression is decreased in OS and its high expression is positively correlated with patient survival. a miR-216b expression was determined by RT-qPCR in OS and paracancerous tissues from patients $(n=60)$, relative to U6. ${ }^{*} p<0.05$ vs. paracancerous tissues by paired $t$-test. b Overall survival in OS patients was determined by the Kaplan-Meier method. c miR-216b expression was determined by RT-qPCR in OS cell lines and normal hFOB1.19 cell line, relative to U6. ${ }^{*} p<0.05$ vs. hFOB1.19 cell line by one-way ANOVA, followed by Tukey's test. Data in panel (b) were compared with unpaired $t$-test. Data are shown as mean \pm standard deviation of three technical replicates 
Table 2 Relationship between clinical characteristics in OS patients and low or high miR-261b expression $(n=60)$

\begin{tabular}{|c|c|c|c|c|}
\hline \multirow[t]{2}{*}{ Demographics } & \multirow[t]{2}{*}{$\mathrm{n}$} & \multicolumn{2}{|c|}{ miR-261b expression (\%) } & \multirow[t]{2}{*}{$P$} \\
\hline & & Low $(n=31)$ & High $(n=29)$ & \\
\hline Sex & & & & 0.456 \\
\hline Male & 40 & 22 & 18 & \\
\hline Female & 20 & 9 & 11 & \\
\hline Age (years) & & & & 0.317 \\
\hline$\leq 20$ & 37 & 21 & 16 & \\
\hline$>20$ & 23 & 10 & 13 & \\
\hline \multicolumn{5}{|l|}{ Location } \\
\hline Femur & 39 & 24 & 20 & 0.459 \\
\hline Others & 21 & 7 & 9 & \\
\hline Postoperative recurrence & & & & $0.001^{*}$ \\
\hline Presence & 44 & 20 & 6 & \\
\hline Absence & 16 & 11 & 23 & \\
\hline Distant metastasis & & & & 0.381 \\
\hline Presence & 22 & 13 & 9 & \\
\hline Absence & 38 & 18 & 20 & \\
\hline
\end{tabular}

* indicates significant difference by chi-square test

fold $=0.974 ;$ SaOS-2 fold $=0.981)$ (Fig. 3a). Furthermore, Pearson correlation analysis highlighted a negative correlation between the expression of miR-216b and JMJD2C in OS tissues (Fig. 3b). In addition, miR-216b agomir was identified to increase the expression of miR$216 \mathrm{~b}$, while reducing the mRNA and protein expression of JMJD2C in MG-63 and SaOS-2 cells (MG63-RTqPCR miR-216b agomir + oe-NC fold $=2.263 ;$ miR-216b agomir + oe-JMJD2C fold $=2.155) \quad($ SaOS-2-RT-qPCR miR-216b agomir + oe-NC fold $=3.043$, miR-216b agomir + oe-JMJD2C fold $=2.955)$ (MG63-western blot analysis miR-216b agomir + oe-NC fold $=0.359 ;$ miR-216b agomir + oe-JMJD2C fold = 3.316) (SaOS-2-western blot analysis miR-216b agomir + oe-NC fold $=0.426$; $\mathrm{miR}$ $216 \mathrm{~b}$ agomir + oe-JMJD2C fold $=2.862$ ) (Fig. 3c). It was further found that miR-216b diminished the cisplatin- induced decrease in cell viability (MG63 0.25 fold $=0.47$, 0.5 fold $=0.45,1$ fold $=0.41) \quad($ SaOS- 20.25 fold $=0.45$, 0.5 fold $=0.41,1$ fold $=0.37$ ), the effect of which was weakened following JMJD2C over-expression (MG63 0.25 fold $=2.55,0.5$ fold $=2.80,1$ fold $=3.68) \quad(\mathrm{SaOS}-2$ 0.25 fold $=2.71,0.5$ fold $=3.24,1$ fold $=3.81$ ) (Fig. 3d). Similarly, miR-216b was observed to enhance cisplatininduced apoptosis (MG63 fold $=2.32 ; \mathrm{SaOS}-2$ fold = $2.73)$, the effect of which was weakened by JMJD2C over-expression $($ MG63 fold $=0.26$; SaOS-2 fold $=0.24$ ) (Fig. 3e). Based on these results, it could be inferred that miR-216b could bind to JMJD2C and inhibit its expressions, ultimately enhancing cisplatin-induced apoptosis in OS cells.

\section{JMJD2C enhances HES1 expression by interacting with HIF1a to remove $\mathrm{H} 3 \mathrm{~K} 9$ methylation modification at the HES1 promoter region in vitro}

In order to characterize the effect of JMJD2C/HIF1 $\alpha$ on HES1, we silenced HIF1 $\alpha$ in cells, and subsequently identified that si-HIF1 $\alpha-3$ exhibited the best HIF $1 \alpha$ silencing efficiency (MG63 si-HIF1 $\alpha-1$ fold $=0.504$, siHIF $1 \alpha-2$ fold $=0.401$, si-HIF $1 \alpha-3$ fold $=0.328) \quad($ SaOS-2 si-HIF $1 \alpha-1$ fold $=0.512$, si-HIF1 $\alpha-2$ fold $=0.392$, siHIF $1 \alpha-3$ fold $=0.250$ ), and was thus chosen for the following experiments (Supplementary Fig. 2). HIF1 $\alpha$ silencing was found to down-regulate the expression of HES1 in MG-63 and SaOS-2 cells (MG63 fold $=0.403$, SaOS-2 fold $=0.445)$ (Fig. 4a). HIF1 $\alpha$ silencing also reversed the elevation in HES1 expression caused by JMJD2C overexpression, demonstrating that HIF $1 \alpha$ functioned as a crucial factor in the up-regulation of HES1 by JMJD2C and HIF1 $\alpha$ (MG63 fold $=2.287$, SaOS-2 fold $=2.260$ ) (Fig. 4b). ChIP-qPCR assay was then employed to detect the quantity of the HES1 promoter in the immune complexes in MG-63 and SaOS-2 cells, the results of which revealed reduced amounts of HES1 promoter in the immune complexes upon JMJD2C over-expression (MG63 fold $=0.13, \mathrm{SaOS}-2$ fold $=0.15$ ), while no significant

Table 3 Univariate and multivariate analyses for predictors of overall survival in OS patients

\begin{tabular}{|c|c|c|c|c|}
\hline \multirow[t]{2}{*}{ Variable } & \multicolumn{2}{|l|}{ Univariate Analysis } & \multicolumn{2}{|l|}{ Multivariate Analysis } \\
\hline & HR $(95 \%$ Cl) & $p$ & HR $(95 \% \mathrm{Cl})$ & $p$ \\
\hline Gender & $0.769(0.316-2.81)$ & 0.562 & $0.804(0.285-2.270)$ & 0.681 \\
\hline Age & $0.800(0.339-1.887)$ & 0.611 & $0.943(0.355-2.507)$ & 0.907 \\
\hline Location & $0.673(0.250-1.814)$ & 0.434 & $0.773(0.274-2.186)$ & 0.628 \\
\hline Distant metastasis & $0.652(0.286-1.488)$ & 0.310 & $0.717(0.297-1.731)$ & 0.459 \\
\hline Histologic grade & $1.348(0.594-3.056)$ & 0.475 & $1.052(0.394-2.806)$ & 0.920 \\
\hline Enneking grade & $1.270(0.557-2.898)$ & 0.570 & $1.124(0.477-2.651)$ & 0.789 \\
\hline miR-216b expression & $0.338(0.139-0.823)$ & $0.017^{*}$ & $0.363(0.142-0.931)$ & $0.035^{*}$ \\
\hline
\end{tabular}

* indicates significant difference 

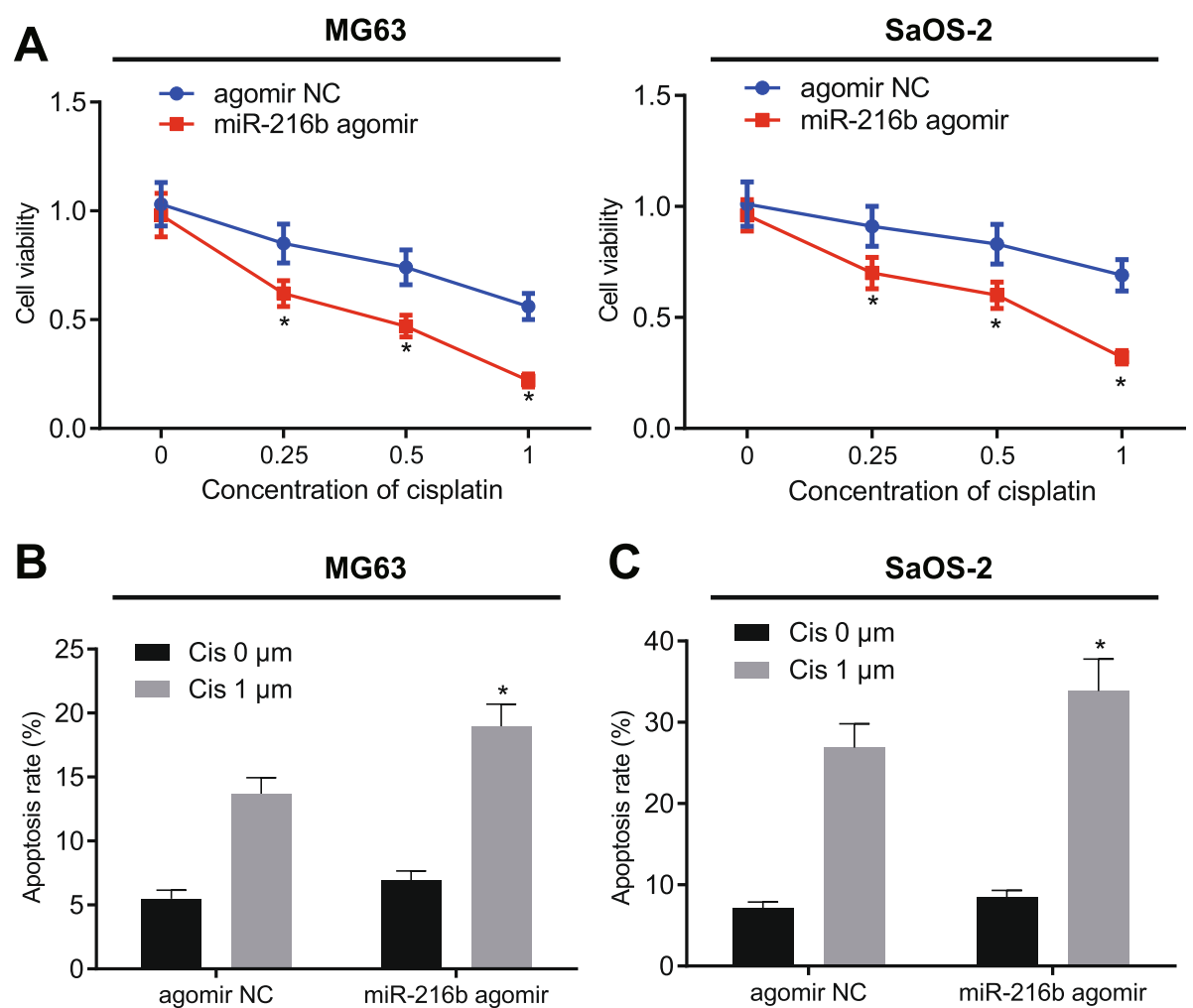

Fig. 2 miR-216b enhances cisplatin-induced apoptosis in OS cells. a Cell viability in MG63 and SaOS-2 cells was determined by CCK-8 assay upon treatment with miR-216b agomir. b Apoptosis of MG63 cells was determined by flow cytometry upon treatment with miR-216b agomir. c Apoptosis of SaOS-2 cells was determined by flow cytometry upon treatment with miR-216b agomir. ${ }^{*} p<0.05$ vs. cells treated with agomir NC. Data in panel $(\mathbf{a})$ and $(\mathbf{c})$ were compared by unpaired t-test while data in panel $(\mathbf{b})$ were compared by repeated measures ANOVA with Bonferroni test. Data are shown as mean \pm standard deviation of three technical replicates

differences were found regarding the quantity of the HES1 promoter (MG63 fold $=1.08$, SaOS-2 fold $=0.93$ ) following combined treatment with oe-JMJD2C and shHIF1 $\alpha(-$ Fig. 4c). Meanwhile, HIF1 $\alpha$ over-expression failed to reverse the down-regulation of HES1 brought about by JMJD2C silencing (MG63-LV-shJMJD2C + oe-NC JMJD2C fold = 0.416, HIF1 $\alpha$ fold $=1.144$, HES1 fold $=0.325)(\mathrm{MG63}-\mathrm{LV}-$ shJMJD2C + oe-HIF1 $\alpha$ JMJD2C fold $=0.742$, HIF1 $\alpha$ fold $=$ 1.908, HES1 fold $=0.820)(\mathrm{SaOS}-2-\mathrm{LV}$-shJMJD2C + oe-NC JMJD2C fold $=0.496$, HIF1 $\alpha$ fold $=1.135$, HES1 fold $=0.396$ ) $(\mathrm{SaOS}-2-\mathrm{LV}$-shJM)D2C + oe-HIF1 $\alpha$ JMJD2C fold $=0.833$, HIF1 $\alpha$ fold $=1.832$, HES1 fold $=0.831$ ) (Fig. 4d). These results demonstrated that JMJD2C and HIF1 $\alpha$ were both essential in the up-regulation of HES1. ChIP-qPCR assay was further applied to detect the quantity of the HES1 promoter enriched by IgG and H3K9me3 antibodies in MG-63 and SaOS- 2 cells, and the results demonstrated that HIF1 $\alpha$ overexpression (MG63 fold $=1.01$, SaOS-2 fold $=0.94$ ) exhibited no effect on the elevated H3K9me3 levels caused by JMJD2C silencing (MG63 fold $=2.61$, SaOS-2 fold $=2.37$ ) (Fig. 4e). In addition, the quantity of HES1 promoter in the immune complexes with anti-H3K9me2 and anti-H3 antibodies was determined in MG-63 and SaOS-2 cells following different treatments (Fig. 4f-g). The quantity of HES1 promoter in the immune complexes with antiH3K9me2 was found to be diminished (MG63 fold $=0.52$, $\mathrm{SaOS}-2$ fold $=0.45$ ) in response to JMJD2C silencing, whereas combined treatment with JMJD2C silencing and HIF1 $\alpha$ over-expression did not negate the effect of JMJD2C silencing (MG63 fold =0.92, SaOS-2 fold=0.89) (Fig. 4f). Besides, JMJD2C silencing and HIF1 $\alpha$ over-expression alone or in combination did not affect the quantity of HES1 promoter in the immune complexes with anti-H3 (MG63 fold = 1.02, SaOS-2 fold=1.04) (Fig. 4g). These findings highlighted the ability of JMJD2C to up-regulate HES1 expression via HIF1 $\alpha$ interaction to diminish H3K9 methylation modification in vitro.

miR-216b enhances cisplatin-induced apoptosis via regulation of the JMJD2C/HIF1a/HES1 signaling axis in OS cells

The transfection of miR-216b agomir and oe-HES1 in MG-63 and SaOS-2 cells was efficient for further experimentation (MG63-RT-qPCR miR-216b agomir + oe-NC fold $=2.315$, miR-216b agomir + oe-HES1 fold $=2.721) \quad(\mathrm{SaOS}-2-\mathrm{RT}-\mathrm{qPCR}$ miR-216b agomir + 


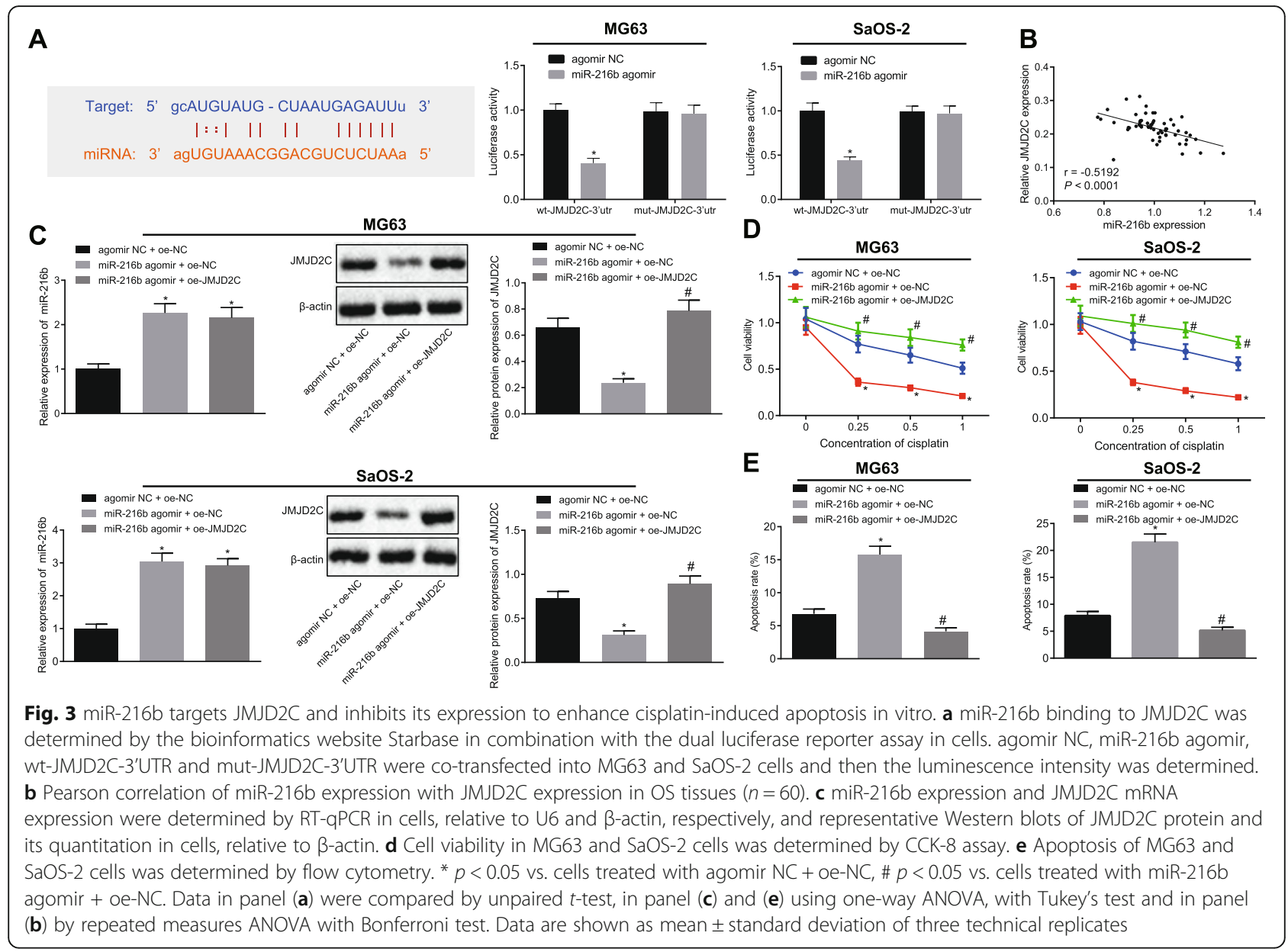

oe-NC fold $=2.845$, miR-216b agomir + oe-HES1 fold = 3.126) $\quad($ MG63-western blot analysis miR-216b agomir + oe-NC fold $=0.222$, miR-216b agomir + oeHES1 fold = 5.532) (SaOS-2-western blot analysis miR-216b agomir + oe-NC fold $=0.259$, miR-216b agomir + oe-HES1 fold=4.310) (Fig. 5a). Overexpression of miR-216b was found to diminish the cisplatin-induced reduction in the viability in MG-63 and SaOS- 2 cells (MG63 0.25 fold $=0.38,0.5$ fold $=$ $0.34, \quad 1$ fold $=0.24), \quad($ SaOS-2 0.25 fold $=0.51, \quad 0.5$ fold $=0.42$, 1 fold $=0.33$ ), the effect of which was abrogated by HES1 over-expression (MG63 0.25 fold = $2.36, \quad 0.5$ fold $=3.04, \quad 1$ fold $=4.02) \quad($ SaOS $-2 \quad 0.25$ fold $=3.32,0.5$ fold $=3.71,1$ fold $=5.20$ ) (Fig. $5 b)$. In addition, miR-216b over-expression further enhanced the cisplatin-induced apoptosis in MG-63 and SaOS2 cells (MG63 fold $=2.76$, SaOS-2 fold = 3.12), whereas HES1 over-expression brought about the opposite effects (MG63 fold $=0.19$, SaOS- 2 fold $=$ 0.25) (Fig. 5c). These results in concert with those discussed above, demonstrated the effect of miR216b/JMJD2C/HIF1 $\alpha /$ HES1 signaling axis on enhancing the effects of chemotherapy in OS cells.
miR-216b enhances the effects of cisplatin via regulation of the JMJD2C/HIF1a/HES1 signaling axis in tumor xenografts of human OS cells

Representative tumor xenografts isolated from nude mice are depicted in Fig. 6a. Tumor growth (day 7 fold = 0.53 , day 14 fold $=0.71$ ) (Fig. $6 \mathrm{~b}$ ) and weight (fold $=$ 0.46) (Fig. 6c) were found to be reduced by cisplatin, while this effect was further increased upon miR-216b over-expression (tumor growth: day 7 fold $=0.69$, day 14 fold $=0.70$ ) (tumor weight: fold $=0.51$ ). Meanwhile, the addition of a HES1 over-expression plasmid weakened the effect of miR-216 over-expression on tumor growth (day 7 fold $=3.30$, day 14 fold $=2.24$ ) and weight (fold $=$ 6.70). Moreover, in tumor tissues, miR-216b expression was observed to be increased, and further increased following miR-216b over-expression. However, no changes were observed in miR-216b expression following the simultaneous over-expression of miR-216b and HES1 in tumor tissues (Fig. 6d). Furthermore, cisplatin was found to diminish the protein expressions of Ki67, Bcl-2, JMJD2C, HIF1 $\alpha$ and HES1 (Ki67 fold $=0.399$, Bcl-2 fold $=0.593$, JMJD2C fold $=0.649$, HIF1 $\alpha$ fold $=0.529$, HES1 fold $=0.547$ ), all of which were further down- 

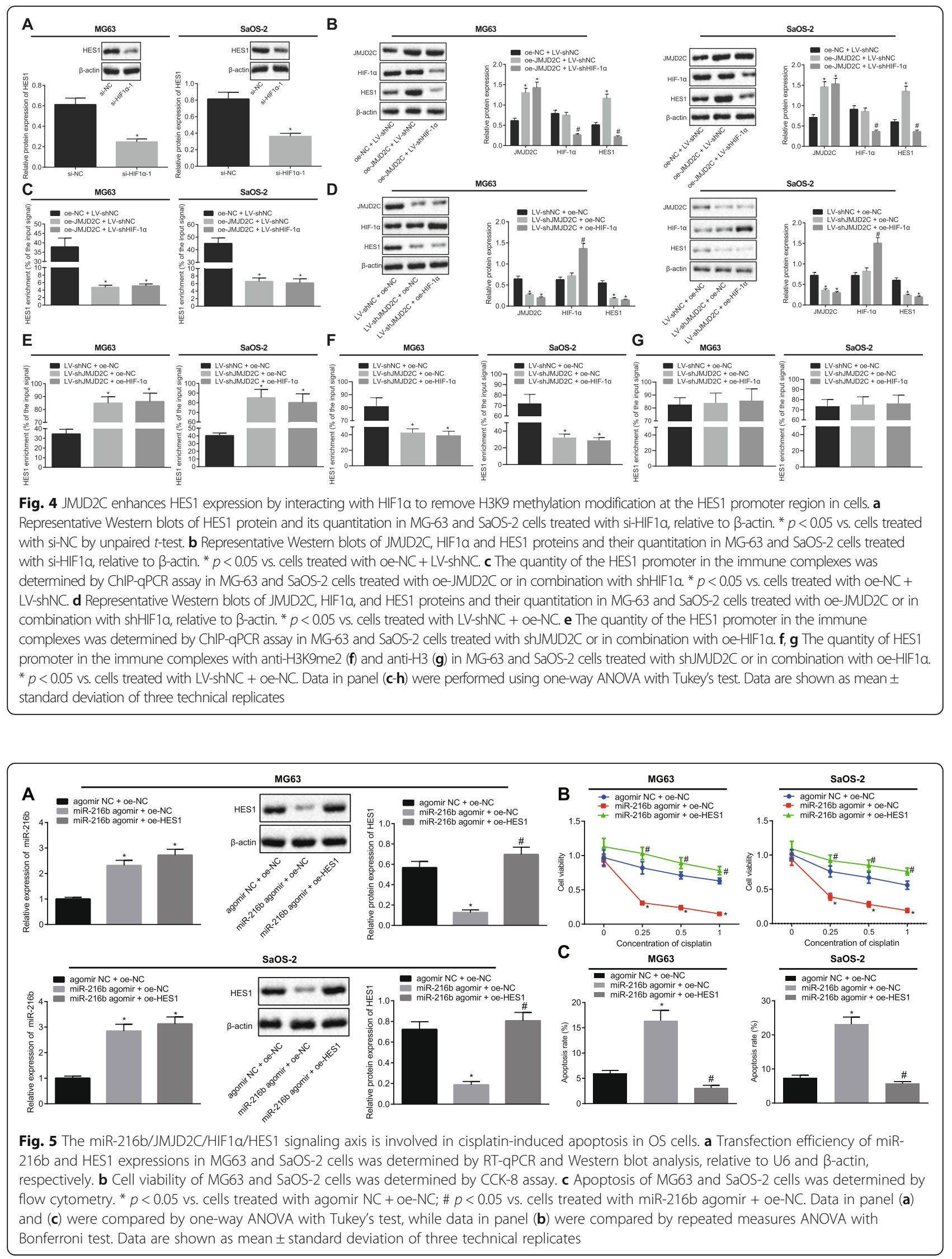


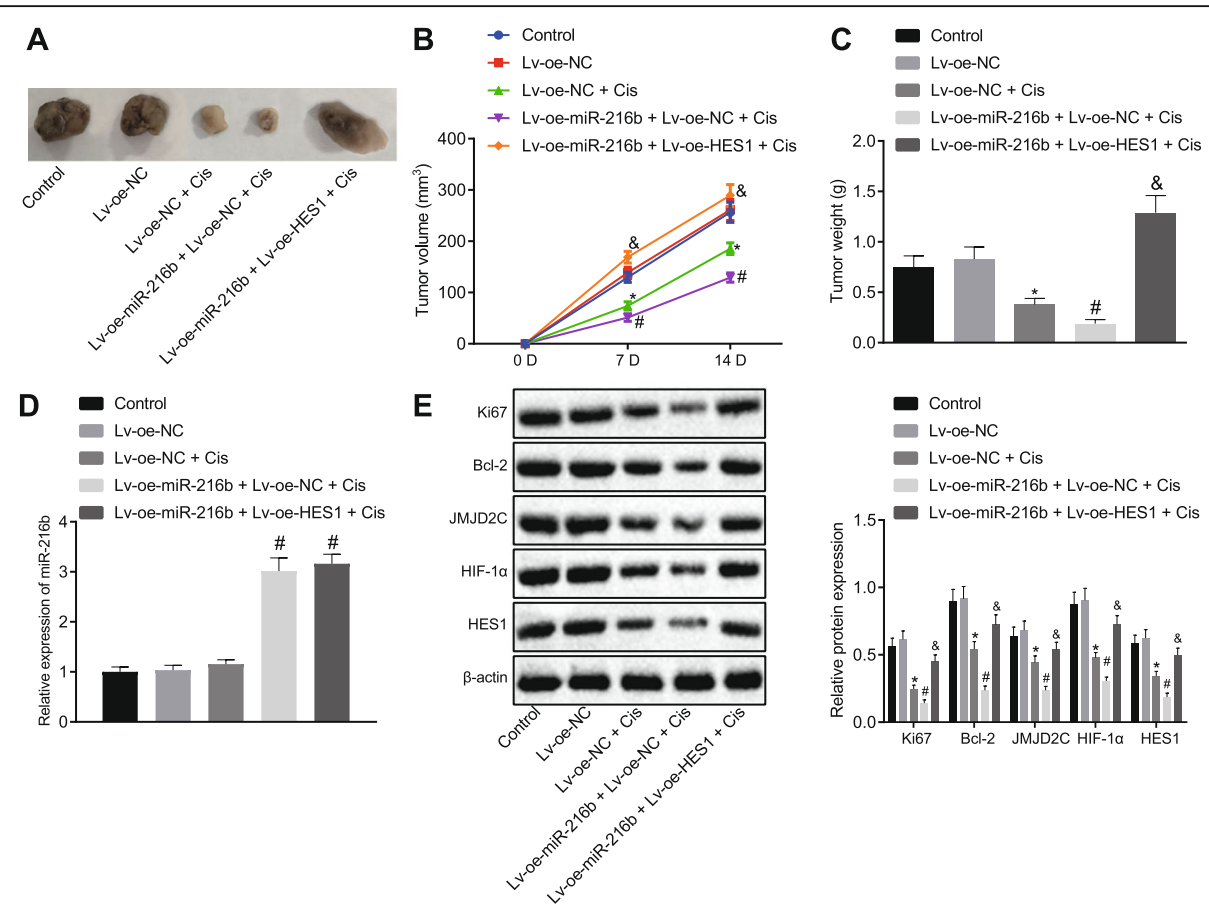

Fig. 6 The miR-216b/JMJD2C/HIF1a/HES1 signaling axis participates in cisplatin-induced apoptosis in vivo. a Representative images of transplanted tumors from nude mice. $\mathbf{b}$ The growth of OS xenograft tumor in nude mice was measured every 7 days. c Tumor weight in nude mice. $\mathbf{d}$ miR-216b expression was determined by RT-qPCR in tumor tissues of nude mice, relative to U6. e Representative Western blots of Ki67, $\mathrm{BCl}-2$, JMJD2C, HIF1a and HES1 proteins and their quantitation in tumor tissues, relative to $\beta$-actin. ${ }^{*} p<0.05$ vs. mice treated with $L v$-oe-NC; \# $p<0.05$ vs. mice treated with Lv-oe-NC + Cis; $\& p<0.05$ vs. mice treated with Lv-oe-miR-216b + Lv-oe-NC + Cis. Data are expressed as mean \pm standard deviation. Data in panel (a) and (c-e) were compared using one-way ANOVA with Tukey's test, while data in panel (b) were compared by repeated measures ANOVA with Bonferroni test. $n=10$ for mice upon each treatment

regulated by miR-216b over-expression $($ Ki67 fold $=$ $0.581, \mathrm{Bcl}-2$ fold $=0.437$, JMJD2C fold $=0.535$, HIF1 $\alpha$ fold $=0.640$, HES1 fold $=0.548$ ) (Fig. 6e). HES1 overexpression was also identified to weaken the effects associated with miR-216b over-expression (Ki67 fold $=3.172$, Bcl-2 fold $=3.051$, JMJD2C fold $=2.274$, HIF1 $\alpha$ fold $=$ 2.373 , HES1 fold $=2.662$ ). These in vivo results provided evidence confirming the promotive role of miR-216b on the effects of cisplatin via the JMJD2C/HIF1 $\alpha / \mathrm{HES} 1$ signaling axis.

\section{Discussion}

Although not as prevalent as many other malignancies, OS represents the foremost primary bone cancer with the vast majority of cases seen among younger populations [1]. In addition, the 5-year survival rate worldwide has failed to improve for decades, remaining between 40 and $70 \%[3-5]$. This can be partly attributed to the emergence of chemotherapeutic resistance in the treatment of OS [37, 38]. Meanwhile, the aberrant regulatory interactions between miRNA-mRNA can potentially mediate the malignant phenotypes of various cancer cells, some of which have shown promise as potential novel targets and therapies aimed at limiting the angiogenesis of malignancies, including OS [39]. Thus, identification of novel non-invasive biomarkers with miRNA-mRNA interaction for the prevention and treatment of OS might prove highly-beneficial. Findings obtained in the current study indicated that miR-216b could augment the effect of the chemotherapeutic agent cisplatin on cell viability and apoptosis in OS cells, which might be attributed to its binding to the JMJD2C gene and the subsequent inhibition of JMJD2C expression, leading to the regulation of the HIF1 $\alpha /$ HES1 signaling axis. Overall, our study demonstrated the role of the JMJD2C/HIF1 $\alpha$ / HES1 signaling axis in the miR-216b-mediated enhancement of the anti-cancer effects of cisplatin.

Firstly, during the course of the current study, we identified that the expression of miR-216b was markedly reduced in OS, whereas a positive correlation was detected between high expression levels of miR-216b and patient survival. Furthermore, our findings revealed that miR-216b could heighten the effect of cisplatin, resulting in reduced OS cell viability and elevated cell apoptosis. Existing literature has demonstrated that miR-216b possesses the ability to directly reduce cancer cell proliferation, migration, and invasion in OS [13]. In addition, another study proposed the use of miR-216b as a 
potential sensitizer in cisplatin chemotherapy owing to its ability to reduce cell viability and promote the apoptosis of cisplatin-resistant ovarian cancer cells [40]. Diminished levels of miR-216b have been detected in nonsmall cell lung cancer cells while enhanced miR-216b expression has been demonstrated to elevate cisplatininduced apoptosis by targeting Beclin-1 [41]. All in all, these findings and evidence suggest that miR-216b could serve as a promising target to enhance the effects of cisplatin on OS cells.

Moreover, miRNAs are known to possess the capacity to modulate gene expressions at a post-transcriptional level by means of interacting with the 3'UTR of specific target mRNAs [42]. During the current study, online biological prediction and luciferase reporter assay were employed, which revealed data indicating that miR-216b could bind to the 3'UTR of the JMJD2C mRNA and negatively-regulate its expression. In line with our findings, JMJD2C has been previously reported to be highlyexpressed in OS, and even capable of elevating the aggressiveness of OS [17]. Therefore, it would be reasonable to speculate that miR-216b could elicit an inhibitory effect on the JMJD2C expression, consequently delaying the progression of OS. Moreover, recent data have evidenced that knockdown of JMJD2A decreases the proliferation of ovarian cancer cells, while simultaneously acting to improve the sensitivity of ovarian cancer cells to cisplatin [43]. The aforementioned findings support the notion that miR-216b binds to JMJD2C and subsequently inhibits its expression, ultimately enhancing cisplatin-induced apoptosis of OS cells.

Furthermore, our findings revealed that JMJD2C upregulated the expression of the HES1 gene via HIF1 $\alpha$ interaction to diminish H3K9 methylation modification. Several studies have stated that HIF1 $\alpha$ is an essential component of JMJD2C functioning [18, 20]. Moreover, HIF1 $\alpha$ and HES1 have been reported to work in tandem to decrease the effects of cisplatin on OS cells, which is very much in line with our findings [22]. As a result, we highlighted JMJD2C and HIF1 $\alpha$ as downstream signaling molecules of miR-216b, wherein miR-216b reduced JMJD2C expression, likely interfering with mRNA stability and JMJD2C action in the demethylation of HES1 gene at the promoter region. More importantly, high expression levels of HES1 have been previously correlated with enhanced OS cell proliferation, migration and invasion as well as boosted chemoresistance [44]. What's more, our findings revealed that miR-216b diminished the expression of HES1 via the removal of H3K9 methylation at the gene promoter site of HES1 in OS cells, which has been previously emphasized as a critical factor for HES1 activation [27]. In contrast, HES1 overexpression weakened the effects of miR-126b, as evidenced by suppressed tumor growth and enhanced cell apoptosis in OS. These results are largely consistent with the previous evidence indicating that HES1 promotes chemotherapy resistance in different cancers $[25,26]$. In addition, we uncovered that HES1 over-expression brought about elevations in the expression of Ki67 and Bcl-2. This is particularly important as Ki67 is recognized as a marker of cancer cell proliferation in OS [45]. Similarly, Bcl-2 is well-documented as a suppressor of apoptosis in various cancers including OS [46]. Consequently, we are convinced that miR-216b may have the potential to enhance cisplatin-induced apoptosis via regulation of the JMJD2C/HIF1 $\alpha / \mathrm{HES} 1$ signaling axis in OS.

\section{Conclusion}

Overall, the current study highlighted that miR-216b augmented cisplatin-induced apoptosis through regulation of the JMJD2C/HIF1 $\alpha / \mathrm{HES} 1$ signaling axis. The newly found miR-216b/JMJD2C/HIF1 $\alpha /$ HES1 signaling axis might pave the way for potential therapeutic mechanisms to reduce chemoresistance in OS. Nevertheless, a few OS cell lines were initially screened in the study, but only MG-63 and SaOS-2 cells were chosen due to highest expression profiles of miR-216b. This selection criterion may pose a selection bias. Therefore, results from our study should be confirmed by future studies incorporating other types of OS cell lines. The current study lends support to the notion that miR-216b may be employed as a chemotherapy adjunct alongside cisplatin for the efficacious treatment of OS.

\section{Supplementary information}

Supplementary information accompanies this paper at https://doi.org/10. 1186/s13046-020-01670-3.

Additional file 1: Supplementary Fig. 1. miR-216b transfection efficiency was determined by RT-qPCR in MG63 and SaOS-2 cells. * $p<0.05$ vs. cells treated with agomir NC by unpaired t-test. Data are shown as mean \pm standard deviation of three technical replicates.

Additional file 2: Supplementary Fig. 2. Silencing efficiency of HIF1a was determined by RT-qPCR in MG-63 and SaOS-2 cells. ${ }^{*} p<0.05$ vs. cells treated with si-NC by unpaired $t$-test.

\section{Abbreviations}

OS: Osteosarcoma; miRs or miRNAs: microRNAs; JMJD2C: Jumonji C domaincontaining 2C; oe: Overexpression; LV: Lentivirus; sh: Short hairpin RNA; NC: Negative control; HIF1a: Hypoxia-inducible factor 1; HES1: Hairy and enhancer of split-1; Co-IP: Co-immunoprecipitation; ChIP: Chromatin immunoprecipitation; DMEM: Dulbecco's modified Eagle's medium; NCBI: National Center for Biotechnology Information; wt: Wild type; mut: Mutant; 3'UTR: 3'untranslated region; CDNA: Complementary DNA; RTqPCR: Reverse transcription quantitative polymerase chain reaction; RIPA: Radio-immunoprecipitation assay; PMSF: Phenylmethanesulfonyl fluoride; BCA: Bicinchoninic acid; SDA-PAGE: Sodium dodecyl sulfate polyacrylamide gel electrophoresis; PVDF: Polyvinylidene fluoride; IgG: Immunoglobulin G; TBST: Tris-buffered saline with Tween-20; HRP: Horseradish peroxidase; ECL: Enhanced chemiluminescence; $\mathrm{BCl}-2$ : B-cell lymphoma 2; CCK-8: Cell counting kit-8; FITC/PI: Fluorescein isothiocyanate/ propidium iodide; ANOVA: Analysis of variance 


\section{Acknowledgements}

We acknowledge and appreciate our colleagues for their valuable suggestions and technical assistance for this study.

\section{Authors' contributions}

Guodong Li and Dong Yang designed the study. Tianyang Xu and Lin Fan collated the data, carried out data analyses and produced the initial draft of the manuscript. Guodong Li and Kaiyuan Liu contributed to drafting the manuscript. All authors have read and approved the final submitted manuscript.

\section{Funding}

This study was supported by General Program of National Natural Science Foundation of China (No. 81572632 \& No. 8187100734).

\section{Availability of data and materials}

The datasets generated/analyzed during the current study are available.

\section{Ethics approval and consent to participate}

The current study was performed with the approval of the ethics committee of Shanghai Tenth People's Hospital (approval number: 2010-0017) and in strict accordance with the Declaration of Helsinki. All participants or their guardians signed informed consent documentation. Animal experiments were performed according to a strictly designed protocol, in accordance with the Guide for the Care and Use of Laboratory Animals published by the US National Institutes of Health. Extensive efforts were made to ensure minimal animal suffering during the study.

\section{Consent for publication}

Not applicable.

\section{Competing interests}

The author declares no competing interest exists.

\section{Received: 11 March 2020 Accepted: 10 August 2020}

Published online: 24 September 2020

\section{References}

1. Caudill JS, Arndt CA. Diagnosis and management of bone malignancy in adolescence. Adolesc Med State Art Rev. 2007;18(1):62-78 ix.

2. Mirabello L, Troisi RJ, Savage SA. Osteosarcoma incidence and survival rates from 1973 to 2004: data from the surveillance, epidemiology, and end results program. Cancer. 2009;115(7):1531-43.

3. Ottaviani G, Jaffe N. The epidemiology of osteosarcoma. Cancer Treat Res. 2009;152:3-13

4. Qiu S, Tao L, Zhu Y. Marital status and survival in osteosarcoma patients: an analysis of the Surveillance, Epidemiology, and End Results (SEER) database. Med Sci Monit. 2019;25:8190-203.

5. Simpson S, Dunning MD, de Brot S, Grau-Roma L, Mongan NP, Rutland CS. Comparative review of human and canine osteosarcoma: morphology, epidemiology, prognosis, treatment and genetics. Acta Vet Scand. 2017 59(1):71.

6. Wang Z, Li S, Li Y, Lin N, Huang X, Liu M, et al. Prognostic factors for survival among patients with primary bone sarcomas of small bones. Cancer Manag Res. 2018;10:1191-9.

7. Kuttesch JF Jr. Multidrug resistance in pediatric oncology. Investig New Drugs. 1996;14(1):55-67.

8. Chou AJ, Gorlick R. Chemotherapy resistance in osteosarcoma: current challenges and future directions. Expert Rev Anticancer Ther. 2006;6(7): 1075-85.

9. Chou AJ, Geller DS, Gorlick R. Therapy for osteosarcoma: where do we go from here? Paediatr Drugs. 2008;10(5):315-27.

10. Li S, Sun W, Wang H, Zuo D, Hua Y, Cai Z. Research progress on the multidrug resistance mechanisms of osteosarcoma chemotherapy and reversal. Tumour Biol. 2015:36(3):1329-38.

11. Palmini G, Marini F, Brandi ML. What is new in the miRNA world regarding osteosarcoma and chondrosarcoma? Molecules. 2017:22(3):417.

12. Di Gregoli K, Jenkins N, Salter R, White S, Newby AC, Johnson JL. MicroRNA24 regulates macrophage behavior and retards atherosclerosis. Arterioscler Thromb Vasc Biol. 2014;34(9):1990-2000.
13. Wang W, Guo Z, Yu H, Fan L. MiR-216b inhibits osteosarcoma cell proliferation, migration, and invasion by targeting Forkhead Box M1. J Cell Biochem. 2019;120(4):5435-43.

14. Huang G, Pan J, Ye Z, Fang B, Cheng W, Cao Z. Overexpression of miR-216b sensitizes NSCLC cells to cisplatin-induced apoptosis by targeting c-Jun. Oncotarget. 2017;8(61):104206-15.

15. Zou J, Kuang W, Hu J, Rao H. miR-216b promotes cell growth and enhances chemosensitivity of colorectal cancer by suppressing PDZ-binding kinase. Biochem Biophys Res Commun. 2017;488(2):247-52.

16. Wu X, Li R, Song Q, Zhang C, Jia R, Han Z, et al. JMJD2C promotes colorectal cancer metastasis via regulating histone methylation of MALAT1 promoter and enhancing beta-catenin signaling pathway. J Exp Clin Cancer Res. 2019;38(1):435.

17. Li X, Dong S. Histone demethylase JMJD2B and JMJD2C induce fibroblast growth factor 2: mediated tumorigenesis of osteosarcoma. Med Oncol. 2015;32(3):53.

18. Luo W, Chang R, Zhong J, Pandey A, Semenza GL. Histone demethylase JMJD2C is a coactivator for hypoxia-inducible factor 1 that is required for breast cancer progression. Proc Natl Acad Sci U S A. 2012;109(49):E3367-76.

19. Contreras-Lopez R, Elizondo-Vega R, Paredes MJ, Luque-Campos N, Torres MJ, Tejedor G, et al. HIF1alpha-dependent metabolic reprogramming governs mesenchymal stem/stromal cell immunoregulatory functions. FASEB J. 2020;34(6):8250-64.

20. El Naggar A, Clarkson P, Zhang F, Mathers J, Tognon C, Sorensen PH. Expression and stability of hypoxia inducible factor 1alpha in osteosarcoma. Pediatr Blood Cancer. 2012;59(7):1215-22.

21. Li T, Xiao Y, Huang T. HIF1alphainduced upregulation of IncRNA UCA promotes cell growth in osteosarcoma by inactivating the PTEN/AKT signaling pathway. Oncol Rep. 2018;39(3):1072-80.

22. Aghazadeh S, Yazdanparast R. Activation of STAT3/HIF-1alpha/Hes-1 axis promotes trastuzumab resistance in HER2-overexpressing breast cancer cells via down-regulation of PTEN. Biochim Biophys Acta Gen Subj. 2017;1861(8): 1970-80.

23. Shi Y, Su Y, Liu C, Tao J, Fu H, Lu C. Inhibition of HES-1 might play a protective role in endothelial cells under cholesterol stimulation via PI3K AKT signaling pathway. Gen Physiol Biophys. 2020:39(2):145-55.

24. Dailey DD, Anfinsen KP, Pfaff LE, Ehrhart EJ, Charles JB, Bonsdorff TB, et al. HES1, a target of Notch signaling, is elevated in canine osteosarcoma, but reduced in the most aggressive tumors. BMC Vet Res. 2013;9:130.

25. Sun L, Ke J, He Z, Chen Z, Huang Q, Ai W, et al. HES1 promotes colorectal cancer cell resistance to 5-Fu by inducing of EMT and ABC transporter proteins. J Cancer. 2017;8(14):2802-8

26. Liu J, Mao Z, Huang J, Xie S, Liu T, Mao Z. Blocking the NOTCH pathway can inhibit the growth of CD133-positive A549 cells and sensitize to chemotherapy. Biochem Biophys Res Commun. 2014:444(4):670-5.

27. Katoh K, Yamazaki R, Onishi A, Sanuki R, Furukawa T. G9a histone methyltransferase activity in retinal progenitors is essential for proper differentiation and survival of mouse retinal cells. J Neurosci. 2012;32(49): 17658-70.

28. Huang J, Ni J, Liu K, Yu Y, Xie M, Kang R, et al. HMGB1 promotes drug resistance in osteosarcoma. Cancer Res. 2012;72(1):230-8.

29. Adnan M, Morton G, Hadi S. Analysis of rpoS and bolA gene expression under various stress-induced environments in planktonic and biofilm phase using 2(-DeltaDeltaCT) method. Mol Cell Biochem. 2011;357(1-2):275-82.

30. Zhou X, Zhang $W$, Jin $M$, Chen J, Xu W, Kong X. IncRNA MIAT functions as a competing endogenous RNA to upregulate DAPK2 by sponging miR-22-3p in diabetic cardiomyopathy. Cell Death Dis. 2017;8(7):e2929.

31. Zhou Z, Wang M, Li J, Xiao M, Chin YE, Cheng J, et al. SUMOylation and SENP3 regulate STAT3 activation in head and neck cancer. Oncogene. 2016; 35(45):5826-38

32. Morovati A, Ahmadian S, Jafary H. Cytotoxic effects and apoptosis induction of cisplatin-loaded iron oxide nanoparticles modified with chitosan in human breast cancer cells. Mol Biol Rep. 2019;46(5):5033-9.

33. Kurokawa $Y$, Sugimoto $N$, Miwa $H$, Tsuda M, Nishina S, Okuda $H$, et al. Phase II study of trastuzumab in combination with S-1 plus cisplatin in HER2positive gastric cancer (HERBIS-1). Br J Cancer. 2014;110(5):1163-8.

34. Naito S, von Eschenbach AC, Giavazzi R, Fidler IJ. Growth and metastasis of tumor cells isolated from a human renal cell carcinoma implanted into different organs of nude mice. Cancer Res. 1986;46(8):4109-15.

35. He C, Sun J, Liu C, Jiang Y, Hao Y. Elevated H3K27me3 levels sensitize osteosarcoma to cisplatin. Clin Epigenetics. 2019;11(1):8. 
36. Li JH, Liu S, Zhou H, Qu LH, Yang JH. starBase v2.0: decoding miRNA-ceRNA, miRNA-ncRNA and protein-RNA interaction networks from large-scale CLIPSeq data. Nucleic Acids Res. 2014;42(Database issue):D92-7.

37. Higuchi T, Oshiro H, Miyake K, Sugisawa N, Han Q, Tan Y, et al. Oral recombinant methioninase, combined with oral caffeine and injected cisplatinum, overcome cisplatinum-resistance and regresses patient-derived orthotopic xenograft model of osteosarcoma. Anticancer Res. 2019;39(9): 4653-7.

38. Higuchi T, Sugisawa N, Miyake K, Oshiro H, Yamamoto N, Hayashi K, et al. Combination treatment with sorafenib and everolimus regresses a doxorubicin-resistant osteosarcoma in a PDOX mouse model. Anticancer Res. 2019;39(9):4781-6.

39. Novello C, Pazzaglia L, Cingolani C, Conti A, Quattrini I, Manara MC, et al. miRNA expression profile in human osteosarcoma: role of miR-1 and miR133b in proliferation and cell cycle control. Int J Oncol. 2013:42(2):667-75.

40. Liu Y, Niu Z, Lin X, Tian Y. MiR-216b increases cisplatin sensitivity in ovarian cancer cells by targeting PARP1. Cancer Gene Ther. 2017;24(5):208-14.

41. Chen L, Han X, Hu Z, Chen L. The PVT1/miR-216b/Beclin-1 regulates cisplatin sensitivity of NSCLC cells via modulating autophagy and apoptosis. Cancer Chemother Pharmacol. 2019;83(5):921-31.

42. Ivey KN, Srivastava D. microRNAs as developmental regulators. Cold Spring Harb Perspect Biol. 2015;7(7):a008144.

43. Zhang H, Wang Z, Wang F, Wang C, Zhang H. IL-6 and IL-8 are involved in JMJD2A-regulated malignancy of ovarian cancer cells. Arch Biochem Biophys. 2020;684:108334.

44. Yu L, Xia K, Gao T, Chen J, Zhang Z, Sun X, et al. The Notch pathway promotes osteosarcoma progression through activation of Ephrin reverse signaling. Mol Cancer Res. 2019;17(12):2383-94

45. Yao W, Wang X, Cai Q, Gao S, Wang J, Zhang P. TRAF4 enhances osteosarcoma cell proliferation and invasion by Akt signaling pathway. Oncol Res. 2014;22(1):21-8.

46. Liu K, Ren T, Huang Y, Sun K, Bao X, Wang S, et al. Apatinib promotes autophagy and apoptosis through VEGFR2/STAT3/BCL-2 signaling in osteosarcoma. Cell Death Dis. 2017;8(8):e3015.

\section{Publisher's Note}

Springer Nature remains neutral with regard to jurisdictional claims in published maps and institutional affiliations.

Ready to submit your research? Choose BMC and benefit from:

- fast, convenient online submission

- thorough peer review by experienced researchers in your field

- rapid publication on acceptance

- support for research data, including large and complex data types

- gold Open Access which fosters wider collaboration and increased citations

- maximum visibility for your research: over $100 \mathrm{M}$ website views per year

At $\mathrm{BMC}$, research is always in progress.

Learn more biomedcentral.com/submissions 